\title{
Mit intranasalem Fentanyl gegen Durchbruchschmerzen?
}

\begin{abstract}
Spontane oder belastungsabhängige Schmerzexazerbationen peinigen $20-95 \%$ der Krebskranken. Fentanyl zeichnet sich als hochpotenter reiner $\mu$-Opioidrezeptoragonist durch einen schnellen Wirkeintritt sowie eine kurze Wirkdauer aus und könnte sich speziell in der intranasalen Form für kurze Schmerzattacken aus heiterem Himmel eignen. Was leistet ein intranasaler Fentanylspray bei den gefürchteten Durchbruchschmerzen, wie sicher und effektiv ist er im klinischen Gebrauch?
\end{abstract}

n einer prospektiven, offenen, nicht interventionellen Multicenter-Studie wurden diese Fragen an opioidgewöhnten Patienten mit Durchbruchschmerzen untersucht, die den Spray im klinischen Alltag über 28 Tage einsetzten und standardisierte Fragebögen und spezielle Tagebücher über ihre Durchbruchschmerzen führten. Insgesamt nahmen 58 deutsche Zentren mit $131 \mathrm{~Pa}$ tienten teil, von denen 116 die Studie beendeten. Ausgewertet wurden 556 Durchbruchschmerzepisoden.

Die 100- $\mu$ g-Dosis wirkte am stärksten und linderte bei $64 \%$ der Patienten die Attacken. $50 \mu \mathrm{g}$ intranasales Fentanyl war bei $28,0 \%$ und $200 \mu \mathrm{g}$ bei $8 \%$ wirksam. Fentanyl besserte die maximalen Durchbruchschmerzen sowohl bei nicht vorbehandelten Patienten als auch bei den 45 Patienten, die mit schnell wirksamen Morphin, transmukosalem Fentanylcitrat oder oralem Dipyrone vorbehandelt waren. Primäre Zielkriterien der Studie von Überall et al. waren die
Schmerzintensität und die Dauer bis zur Schmerzlinderung. Die Patienten gaben eine Schmerzlinderung bei $81,9 \%$ aller Episoden innerhalb von 5 Minuten an. Nach 10 Minuten gaben 94,3\% eine analgetische Wirkung an und bei $81,4 \%$ war sie bereits maximal. Durch diese schnelle Analgesie stieg die Lebensqualität und Einschränkungen im Alltag nahmen ab. Ärztliche Interventionen oder Einsätze des Pflegedienstes sowie des ambulanten Palliativdienstes wurden seltener nötig. Das intranasale Fentanly wurde gut vertragen, nur sechs Patienten klagten über eine Nebenwirkung (4,6\%) wie Benommenheit, Erbrechen und Müdigkeit. Alle Nebenwirkungen waren nur mäßig ausgeprägt und lösten sich ohne Therapie. Von den vorbehandelten Patienten gaben 96,2\% an, dass die intranasale Fentanyltherapie schneller und bei $81 \%$ stärker analgetisch wirkte. $77 \%$ fanden sie besser verträglich und $69 \%$ auch einfacher $\mathrm{zu}$ handhaben.
Fazit: Intranasales Fentanyl stellt ein hocheffektives und verträgliches Analgetikum gegen Durchbruchschmerzen dar. Sowohl die Krebskranken als auch ihre Behandler beurteilten diese Therapieform als schnell wirksam, effektiv und gut verträglich. Stephanie Kraus

Überall MA et al. Efficiency of Intranasal Fentanyl in Patients with Breakthrough Cancer Pain in Daily Practice - Results of the German NonInterventional Study with Instanyl ${ }^{\circledR}$ (GENISIS). Glob J Anesthesiol. 2014; 1 (1): 104

\section{Kommentar von}

\section{Dr. Johannes Horlemann}

Das Opioid "Fentanyl“ gilt leitliniengerecht als das geeignete Medikament gegen Durchbruchschmerzen. Solche Schmerzen setzen eine stabile Hintergrundeinstellung mit einem Opioid voraus. Die Deutsche Multicenterstudie GENISIS von Überall et al. untersuchte den intranasalen Applikationsweg auf Effektivität und Verträglichkeit. Nahezu alle Patienten waren nach 5 oder 10 Minuten nach Anwendung des Sprays schmerzfrei. Nebenwirkungen waren selten. Intranasales Fentanyl, vorzugsweise bei der Dosis von $100 \mathrm{mg}$, sichert daher Lebensqualität und entlastet die Behandler.

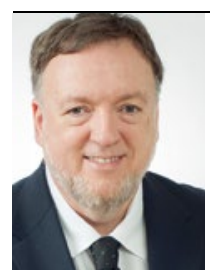

Dr. med. Johannes Horlemann, Kevelaer Facharzt für Allgemeinmedizin, spezielle Schmerztherapie, Leiter des Regionalen Schmerzzentrums DGS Geldern

\section{Opioid-Langzeittherapie wird für viele zum Problem}

\section{Eigentlich sollten Opioide für nicht maligne Schmerzen möglichst nicht dauerhaft gegeben werden. Nach Daten eines norwegischen Registers bleibt jedoch jeder Vierte mit einer Langzeittherapie an der Medikation hängen.}

erade angesichts vieler Todesfälle durch Opioidmissbrauch in den USA wird diskutiert, wer wie lange solche Analgetika erhalten sollte. Da es zur Praxis in Deutschland kaum verlässliche Daten gibt, lohnt sich ein Blick nach Skandinavien. So werden etwa in Nor- wegen sämtliche Arzneimittelverordnungen zentral in einem Register erfasst. Dieses haben sich nun Schmerzforscher von der Universität in Trondheim zunutze gemacht. Sie schauten, wer alles im Jahr 2005 Opioide bekam und wer von diesen Patienten auch noch in den fol- genden sechs Jahren solche Schmerzmittel benötigte. Insgesamt konnten sie in der Datenbank rund 417.000 Patienten aufspüren, die im Jahr 2005 Opioide aufgrund von chronischen, nicht tumorbedingten Schmerzen (CNTS) erhalten hatten - das sind $12 \%$ der Bevölkerung. Davon bekam wiederum fast jeder Zehnte eine Langzeitbehandlung mit Opioiden, definiert als mehr als 180 Tagesdosen pro Jahr. Mehr als $1 \%$ der Bevölkerung wurde also langfristig aufgrund von CNTS mit Opioiden behandelt.

Von den rund 45.000 Personen mit Opioid-Langzeittherapie im Jahr 2005 waren $39 \%$ erstmals in diesem Jahr mit 